\title{
ACCORD: Schwere Hypoglykämien erhöhen Mortalität
}

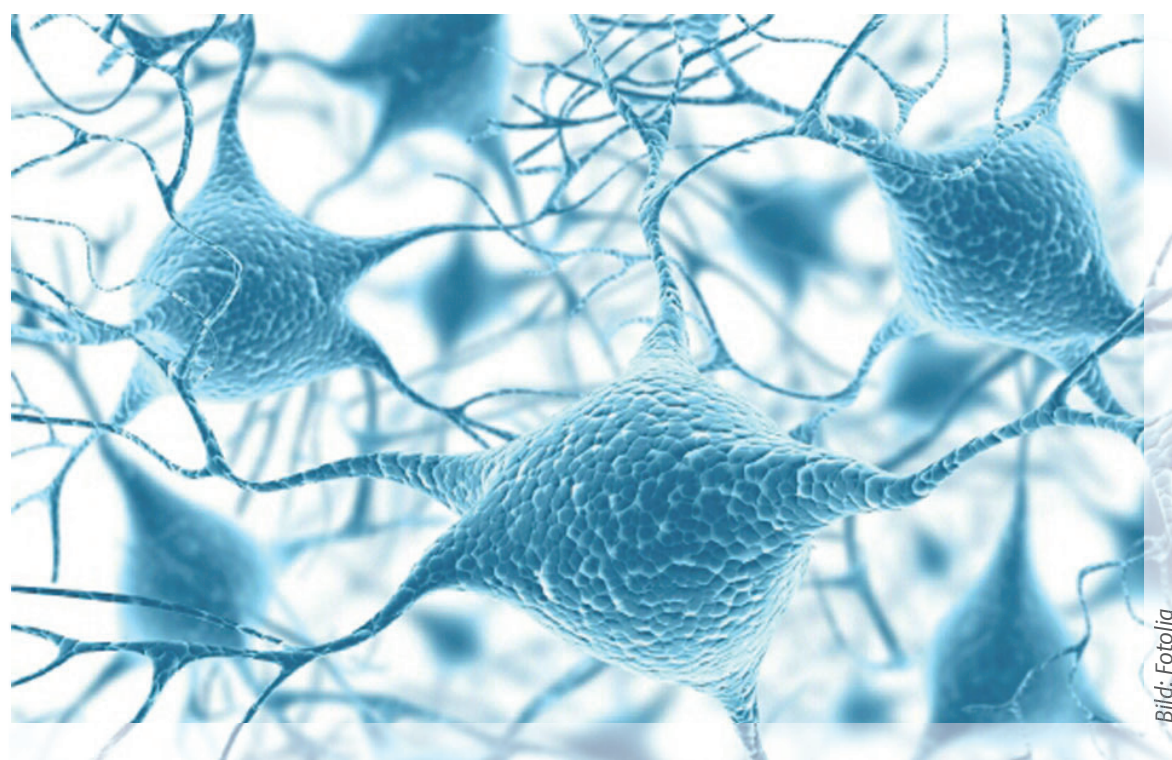

Hintergrund: In der ACCORD-Studie führte die Absenkung der $\mathrm{HbA}_{1 \mathrm{c}}$-Werte auf ungefähr $6,5 \%$ zu einer signifikant höheren Mortalität im Vergleich zu einer Absenkung auf $\mathrm{HbA}_{1 \mathrm{c}}$-Werte von ungefähr $7,5 \%$. In einer jetzt publizierten retrospektiven Analyse waren symptomatische schwere Hypoglykämien mit einer erhöhten Mortalitätsrate assoziiert. Methode: In die Studie Action to Control Cardiovascular Risk in Diabetes (ACCORD) wurden Typ-2-Diabetiker mit $\mathrm{HbA}_{1 \mathrm{c}}$-Werten von 7,5\% und höher eingeschlossen, welche entweder im Alter von 40-79 Jahre waren und eine vorbestehende kardiovaskuläre Erkrankung hatten oder im Alter von 55-79 Jahre waren und eine subklinische kardiovaskuläre Erkrankung oder mindestens 2 weitere kardiovaskuläre Risikofaktoren hatten. Der Ziel-HbA in der intensiv behandelten Gruppe sollte unter $6,0 \%$ und in der Standardgruppe zwischen 7,0 und 7,9\% liegen. Erfasst wurden symptomatische schwere Hypoglykämien mit entweder gemessenen Blutglukosewerten unter $50 \mathrm{mg} / \mathrm{dl}(2,8 \mathrm{mmol} / \mathrm{l})$ oder solche, bei welchen die Hilfe einer weiteren Person oder durch medizinisches Personal erforderlich war, sowie die Gesamtmortalität und morbiditätsbezogene Mortalitätsraten.

Ergebnisse: Daten zu Hypoglykämien lagen bei 10194 der insgesamt 10251 ACCORD-Teilnehmer vor. Die jährliche Inzidenz an Hypoglykämien betrug in der intensiv behandelten Gruppe 3,14\% und in der Standardgruppe 1,03\%. Die Hypoglykämierate war signifikant erhöht für Frauen $(p=0,03)$, Afroamerikaner ( $p<0,0001$ im Vergleich zu Kaukasiern), Personen ohne Schulabschluss $(p<0,05$ im Vergleich zu Personen mit Hochschulabschluss), ältere Personen ( $\mathrm{p}<0,0001$ pro Lebensjahr) und bei Insulintherapie $\mathrm{zu}$ Studienbeginn $(\mathrm{p}<0,0001)$.

Jede Absenkung der $\mathrm{HbA}_{1 \mathrm{c}}$-Werte in den ersten 4 Studienmonaten um einen Prozentpunkt war mit einer Risikoreduktion um $28 \%$ in der Standardgruppe (19-37\%) bzw. $14 \%$ in der intensiv behandelten Gruppe (4-23\%) für Hypoglykämien mit Fremdhilfe durch medizinisches Personal assoziiert. Demgegenüber erhöhte sich das Risiko für Hypoglykämien mit Fremdhilfe durch medizinisches Personal mit jeder $1 \%$ igen $\mathrm{HbA}_{1 \mathrm{c}}$-Wert-Senkung im weiteren Studienverlauf, und zwar sowohl in der Standardgruppe (Hazard ratio (HR) 1,76; 95\%-KI 1,50-2,06) als auch in der intensiv behandelten Gruppe (HR 1,15; 95\%-KI 1,02-1,21).

Die adjustierte jährliche Mortalitätsrate in der intensiv behandelten Gruppe betrug 2,8\% für diejenigen, die eine oder mehrere schwere Hypoglykämie-Episoden hatten, im Vergleich zu 1,2\% bei denjenigen ohne schwere Hypoglykämien (53 Todesfälle pro 1924 Personenjahren vs. 201 Todesfällen pro 16315 Personenjahren; adjustierter HR 1,41; 95\%-KI
1,03-1,93). Ein ähnlicher Zusammenhang wurde in der Standardgruppe beobachtet: Die adjustierte jährliche Mortalitätsrate betrug hier bei Personen mit schweren Hypoglykämien 3,7\% (21 Todesfälle pro 564 Personenjahre) versus 1,0\% (176 Todesfälle pro 17297 Personenjahre; adjustierter HR 2,30; 95\%-KI 1,46-3,65). Andererseits war bei Personen mit mindestens einer schweren Hypoglykämie in der intensiv behandelten Gruppe das Mortalitätsrisiko (nicht signifikant) geringer als in der Kontrollgruppe (adjustierter HR 0,74; 95\%-KI 0,46-1,23). Der Mortalitätsvorteil zugunsten der intensiv behandelten Personen war sogar signifikant, wenn Personen mit Hypoglykämien und Fremdhilfe durch medizinisches Personal beurteilt wurden (adjustierter HR 0,55; 95\%-KI 0,31-0,99). Von den 451 Todesfällen, die sich in ACCORD bis zu dem Zeitpunkt, an dem die intensive Behandlung beendet werden musste, ereigneten, war lediglich ein Todesfall sicher mit einer Hypoglykämie in Verbindung zu bringen.

Folgerung: In ACCORD war bei Patienten mit hohen $\mathrm{HbA}_{1 c}$-Werten das Hypoglykämierisiko erhöht. Die zu Studienbeginn erreichte rasche $\mathrm{HbA}_{1 \mathrm{c}}$-Senkung führte nicht zu einem Anstieg des Hypoglykämierisikos. Symptomatische schwere Hypoglykämien waren mit einer erhöhten Mortalitätsrate sowohl in der intensiv behandelten Gruppe als auch in der Standardgruppe assoziiert. Allerdings fand sich bei Personen mit schweren Hypoglykämien eine geringere Mortalitätsrate in der intensiv behandelten Gruppe im Vergleich zur Standardgruppe. Schwere Hypoglykämien sind nicht verantwortlich für die unterschiedliche Mortalitätsrate zwischen den beiden ACCORD-Studienarmen, so die Autoren.

Dr. med. Winfried Keuthage, Münster

Quellen: Miller ME et al. The effects of baseline characteristics, glycaemia treatment approach, and glycated haemoglobin concentration on the risk of severe hypoglycaemia: post hoc epidemiological analysis of the ACCORD study. BMJ 2010; 340: b4909 online

Bonds DE et al. The association between symptomatic, severe hypoglycaemia and mortality in type 2 diabetics: retrospective epidemiological analysis of the ACCORD study. BM] 2010; 340: b5444 online 